\title{
Optimization of equipment deployment on fire trucks
}

\author{
Tadeusz Nowicki ${ }^{1, *}$ \\ ${ }^{1}$ Military University of Technology, Cybernetics Faculty, 00-908 Warsaw, gen. S. Kaliskiego str., Poland
}

\begin{abstract}
In this work, the problem of deploying firemen' equipment is discussed in order to achieve the best value of ergonomic indicators for fireman during anti-fire action. The equipment can be deployed on the fore truck in a different ways. We are looking for the best way to do it from the point of view of ergonomics. The multi-criterial optimization problem of equipment location on the vehicle has been formulated. It is linear integer programming problem with three criteria functions. It is able to solve that problem using formal method after some modifications. It is possible to receive several solutions from multi-criterial optimization problem and then evaluate them in a virtual simulation environment. It can then be taken into account a number of additional factors that are important for fireman and ergonomics.
\end{abstract}

\section{Introduction}

The issues of ergonomics in the design of technical objects are often connected with numerous problems in different applications $[1,2,3]$. If they are related to staff serving technical equipment, there are problems in the sphere of discussion:

- empirical study of human work in concrete conditions of material work environment,

- creating guidelines for new (modernized) designers, technologies, products,

- setting criteria for assessing working conditions,

- to support a systematic study of the health of employees,

- development of standards, measures and undertakings ensuring optimal working conditions,

- supporting activities aimed not only at adapting to the conditions of so called "material working environment" to man but also man to work under these conditions,

- provision of the basis for the prevention of occupational disease, including industrial poisoning, in the prevention of occupational disease prevention.

In the event of a problem with the deployment of equipment in a rescue and fire fighting vehicle taken into account, some of the above points shall be taken into account.

The development of standards, equipment and activities to optimize the working conditions is to determine the equipment to be deployed in the rescue and fire fighting vehicle and to deploy it in the useable recesses of rescue and firefighting vehicles designed for this purpose. The result of this study will be the guidelines for designers equipping new or upgraded fire trucks.

In the issue of deployment of equipment rescue and firefighting vehicles are guided by the working conditions of persons - the personnel of the vehicle. A number of factors are taken into account, but the problem is primarily dedicated to take into account ergonomic factors. It should be emphasized that these factors are particularly important in multiple actions in a relatively short time and refer to the same group of people. Possible effects of misplaced equipment in a rescue and fire fighting vehicle will be cumulatively accumulated to the health of the crew members of the vehicle. The definition of criteria for evaluating working conditions will be mainly related to the ergonomics of firefighters taking part in rescue operations.

The problem of empirical study of human work in concrete conditions of material work environment [9][14] is not easy to solve in a short time. It would be necessary to carry out a number of specialized personnel surveys for various equipment installations in rescue and firefighting vehicles in a large sample of various types of rescue and firefighting operations. This would not only be a long-term undertaking but would also add to the health of the staff. So decided to solve quite innovative. Computer simulations of rescue personnel actions for various types of actions will be used to simulate the various deployment variants on the vehicle in terms of ergonomics.

From the labor ergonomics point of view, the following factors are taken into account in the analysis of the human factor load:

- physical load,

- psychological load,

- environmental load,

- organizational load.

The order of the loads is not accidental here. The most important thing is physical stress, which is independent of others and is a constant problem in different types of actions. Its positive change is essential if it is possible. Other loads can be adjusted by training, treatment, organizational regulations, etc. 
Therefore, workload will be considered physical and to some extent organizational. Criteria for taking into account the physical strain in the process of unloading equipment from a rescue and fire fighting vehicle for various types of rescue rescue operations, however, must be remembered that physical effort and the effects of rescue personnel work differ significantly in the vertical movement of equipment (taking off equipment from fire truck) and horizontal relocation of equipment to the place of its distribution in the rescue and firefighting operation.

Next, this time about the organizational nature of the criterion will be the time of unloading equipment at the beginning of the rescue and firefighting operation. On the one hand, the short time of unloading of equipment is a factor which additionally loads a crew member of a rescue and firefighting vehicle and, on the other hand, is an important element of a smooth operation of this type. A decision has been made to include this criterion in assessing the ergonomics of deploying equipment in a rescue and fire fighting vehicle.

You can specify a number of assumptions and requirements for the deployment problem (in the literature also referred to as localization or packing problem) equipment on a fire brigade vehicle:

- one of the problems is determining whether the equipment is to be positioned high or low in modular vehicle construction,

- another problem is whether certain sets of equipment are to be close to each other or whether they can be placed at a relative distance,

- another problem is whether certain sets of equipment are to be placed on one side of the vehicle or can be placed on two other sides of the vehicle,

- in principle, the rear of the vehicle is defined as it is an accessory for the control of water and foam feeders, etc.,

- another problem is whether certain sets of equipment are to be placed deep or shallow in the modular construction of the vehicle,

- it is important that the weight distribution of the fire truck is taken into account, as poor weight distribution can cause accidents during rapid maneuvering of vehicles on the road or in the field during various difficult maneuvers when the crew tries to position the vehicle at a suitable location,

- sprinklers used in vehicles during firefighting operations should also be considered in the problem of location of equipment on fire brigade vehicles,

- crew safety elements are dependent on: environmental conditions of the operation (icing, snow, rain, mud, etc.), order of firefighting equipment (sequence of actions to be determined), personnel load during firefighting equipment (load musculoskeletal system),

- cars should stand outside the danger zone, so at a distance from the target object, so the placement of certain equipment should take into account that fact,

- it is necessary to use roller shutters with safety belts, so packing should take into account the profile and limitations of the vehicle's construction using rescue and fire-fighting equipment.

\section{Location of equipment in fire trucks}

From the point of view of the manufacturer of rescue and firefighting vehicles, equipment used for rescue and firefighting operations can be deployed in the recesses of fire trucks as shown in the figure below.
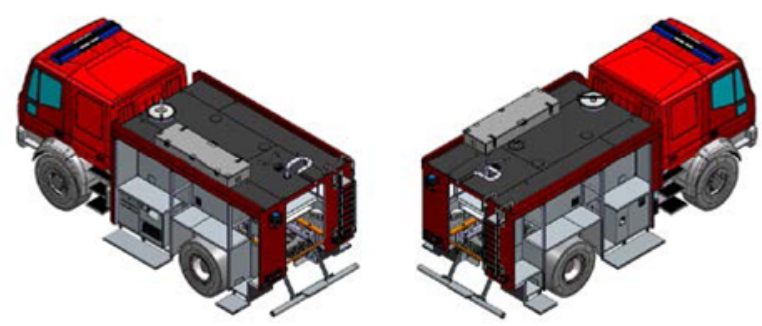

Fig. 1. Possible location of equipment on fire trucks.

As a result of these considerations we have the situation that rescue and firefighting equipment can be placed in recesses on both sides of the vehicle. But these are not many. The following two drawings (Figure 3 and Figure 4) show the recesses that may be considered in the deployment of rescue and firefighting equipment on the sides of the rescue and fire fighting vehicle.

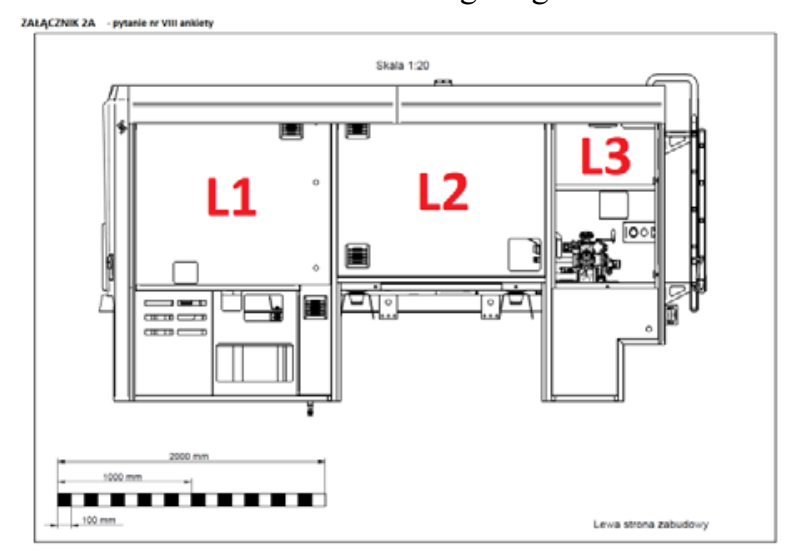

Fig. 2. Possible location of equipment on left side of fire trucks.

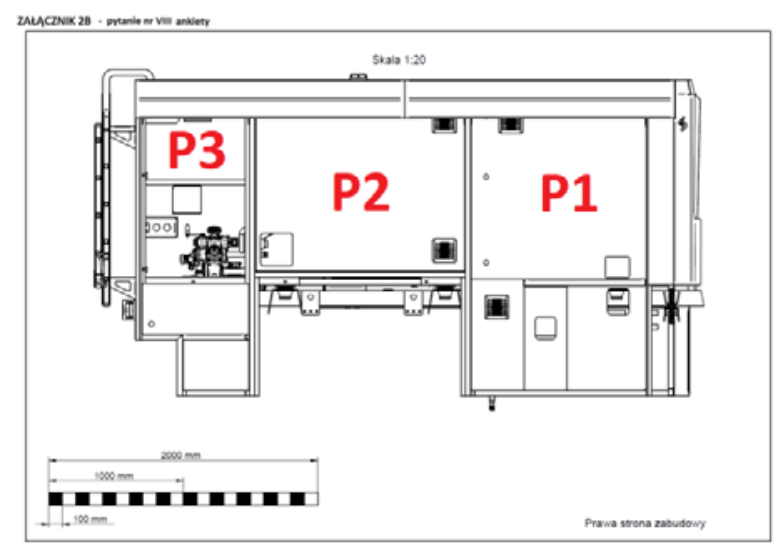

Fig. 3. Possible location of equipment on right side of fire trucks. 


\section{The problem of equipment location in fire trucks}

It can be assumed that in the course of subsequent arrangements of the project team the set of equipment for deployment in the recesses of the rescue and firefighting vehicle (specified in special duty regulations) may change.

Assume, therefore, that we are dealing with a set of $\mathrm{N}$ elements of rescue and firefighting equipment whose set of numbers is defined below:

$$
\mathrm{S}=\{1,2,3, \ldots, n, \ldots, N\}
$$

Number $\mathrm{N}$ is currently specified. For each of these elements of rescue and firefighting equipment, its characteristics are:

$r_{n}$ - spatial size of $n$-th element of rescue and firefighting equipment,

$c_{n}$ - weight of $n$-th element of rescue and fire-fighting equipment,

$d_{n}$ - mean distance from the rescue and fire-fighting vehicle in which the n-th element of rescue and firefighting equipment should be placed,

$t_{n}$ - time needed for displacement of $n$-th element of rescue and firefighting equipment at distance $d_{n}$.

Rescue and fire-extinguishing equipment can not be deployed in full freedom from the point of view of its use in rescue and firefighting operations. Certain pieces of equipment are grouped into kits in which the equipment must be placed in the recesses of the rescue and fire fighting vehicle side by side.

Assume that there are known kits (alternatively - sets) of rescue and firefighting equipment related to each other from a functional point of view. Let's assume that number of these sets is $\mathrm{K}$. The sets of sets numbers are defined as follows:

$$
Z=\{1,2,3, \ldots, k, \ldots, K\}
$$

Each kit is defined by the elements of the primary components of rescue and firefighting equipment. The formal description of the sets is as follows:

$$
Z_{k}=\{n \in S: \text { selected items from the set } S\}
$$

These kits meet the following conditions:

$$
Z_{k} \cap Z_{i}=\varnothing, i, j=1, \ldots, N, i \neq j
$$

and

$$
\bigcup_{k \in Z} Z_{k}=S
$$

Then the characteristics of the individual sets have the figures given below in the correct order with regard to the definition of the components of rescue and firefighting equipment. Total set size is:

$$
r^{k}=\sum_{n \in Z_{k}} r_{n}
$$

Total weight of the set is:

$$
c^{k}=\sum_{n \in Z_{k}} c_{n}
$$

Whereas the average distance from the rescue and firefighting vehicle in which the elements of the rescue and fire fighting equipment included in the $\mathrm{k}$-th set of the formal average are to be placed:

$$
d^{k}=\frac{1}{\overline{\overline{Z_{k}}}} \sum_{n \in Z_{k}} d_{n} \text {, }
$$

Also, recount the niches in the rescue and extinguishing vehicle. There are six, so the first three to the left are numbered from 1 to 3 , while the right numbered from 4 to 6. If there are additional spaces from the beech or the roof of the vehicle, let's suppose that these places will be J. Hence, the set of numbers of considered recesses in a rescue vehicle for use is defined below:

$$
M=\{1,2,3, \ldots, j, \ldots,, J\}
$$

For each of these recesses, the following characteristics are defined:

$\mathrm{p}^{\mathrm{j}}$ - spatial volume of the jth cavity of the rescue and firefighting vehicle,

$\mathrm{w}^{\mathrm{j}}$ - average height from the ground or from the shelf on

which the vehicle personnel can stand against the $\mathrm{j}$-th recess of the rescue and fire-fighting vehicle.

The following problem arises: we are looking for the deployment of rescue and firefighting equipment in the recesses of the rescue and firefighting vehicle, which is the decision matrix $\mathrm{X}$ :

$$
X=\left\lfloor x_{k j}\right\rfloor_{K x J}
$$

such that $\mathrm{x}_{\mathrm{kj}}$ - is equal to 1 if the $\mathrm{k}$-th set of rescue and firefighting equipment is placed in the $\mathrm{j}$-box (recess) or equals 0 otherwise.

Circumstances for elements of matrix $\mathrm{X}$ are given below:

$$
x_{k j} \in\{0,1\}, k=1, \ldots, K, j=1, \ldots, J
$$

this means that the decision variables are binary variables

$$
\sum_{j=1}^{J} x_{k j}=1, k=1, \ldots, K
$$

that means that each kit must be in one of the recesses of the rescue and fire-fighting vehicle, and in addition only one,

$$
\sum_{k=1}^{K} x_{k j} \cdot r^{k} \leq p_{j}, j=1, \ldots, J
$$

that means that the capacity of the kits placed in each of the recesses cannot exceed its total capacity and

$$
\left|\sum_{k=1,2,3} c^{k}-\sum_{k=4,5,6} c^{k}\right| \leq \varepsilon
$$

This means that the weight load on both sides of the vehicle axle is smaller than the assumed magnitude $\varepsilon$ of a practically determined magnitude, so that the vehicle 
will not have a tendency to tilt while crossing corridors on access roads to the rescue and firefighting site. The last restriction is nonlinear. However, it can be shown that after inserting auxiliary variables this inequality can be reduced to a linear form.

The function of the criterion of distribution of rescue and firefighting kits in fire truck is vector:

$$
F(X)=\left(F_{1}(x), F_{2}(X), F_{3}(X)\right)
$$

where

$$
F_{1}(X)=\sum_{j=1}^{J} \sum_{k=1}^{K} x_{j k} \cdot c^{k} \cdot w^{j}
$$

means the total weight of vertical displacement of rescue and fire-fighting equipment during the action,

$$
F_{2}(X)=\sum_{j=1}^{J} \sum_{k=1}^{K} x_{j k} \cdot c^{k} \cdot d^{k}
$$

means the total weight of horizontal displacement of rescue and firefighting equipment during the action,

$$
F_{3}(X)=\sum_{j=1}^{J} \sum_{k=1}^{K} x_{j k} \cdot \sum_{n \in Z_{k}} t_{n}
$$

means the total time of moving rescue and firefighting equipment during the action.

Thus, the problem of multi-criterion minimization is in following form: to choose such $\mathrm{X}$ * to minimize

$$
\begin{gathered}
F_{1}\left(X^{*}\right)=\min _{X \in D} \sum_{j=1}^{J} \sum_{k=1}^{K} x_{j k} \cdot c^{k} \cdot w^{j} \\
F_{2}\left(X^{*}\right)=\min _{X \in D} \sum_{j=1}^{J} \sum_{k=1}^{K} x_{j k} \cdot c^{k} \cdot d^{k} \\
F_{3}\left(X^{*}\right)=\min _{X \in D} \sum_{j=1}^{J} \sum_{k=1}^{K} x_{j k} \cdot \sum_{n \in Z_{k}} t_{n}
\end{gathered}
$$

where the set $\mathrm{D}$ of acceptable solutions takes the form:

$$
\begin{gathered}
x_{k j} \in\{0,1\}, k=1, \ldots, K, j=1, \ldots, J \\
\sum_{j=1}^{J} x_{k j}=1, k=1, \ldots, K \\
\sum_{k=1}^{K} x_{k j} \cdot r^{k} \leq p_{j}, j=1, \ldots, J \\
\left|\sum_{k=1,2,3} c^{k}-\sum_{k=4,5,6} c^{k}\right| \leq \varepsilon
\end{gathered}
$$

As a result, there is the problem of multi-criterion linear binary mathematical programming $[4,5,6,7]$, which can be solved by various methods of multicriterial linear programming [7].
It is worth noticing, that this problem can be decomposed into a sequence of detailed tasks with single criteria:

- what size and where to deploy the modules in the fire brigade vehicle (unless you have already specified where and what to be),

- how to arrange certain sets of firefighting equipment on the vehicle in the fire engine vehicle modules,

- at the same time: how to arrange certain kit (s) of rescue equipment on the vehicle in the fire engine vehicle modules.

taking into account also:

- the frequency of various types of firefighting actions,

- sequences and sequences of actions performed in different types of firefighting actions,

- sets of equipment and elements used in different types of firefighting actions.

The frequency of various types of firefighting activities can be derived from the studies that determine the type of equipment and how often it is used in certain types of fire operations. These studies have been conducted for about a year and their results will be achievable in the near future.

Then let vector A denote the number of fire action types:

$$
A=\{1,2,3, \ldots, i, \ldots, I\}
$$

where I is the number of different types of rescue and firefighting operations.

We also assume that the numbers of rescue firefighting kits used in each type of firefighting action are known. Let the set of rescue and firefighting kit numbers used in the $\mathrm{i}$-th rescue and firefighting operations take the form:

$$
N^{i}=\{k \in S: \text { selected elements from the set } \mathrm{S}\}
$$

where $\mathrm{i}=1, \ldots, \mathrm{I}$.

In turn, the frequency of occurrence of particular types of actions, learned from the research defined by the vector:

$$
f=\left(f_{1}, f_{2,} f_{3}, \ldots, f_{i}, \ldots, f_{I}\right)
$$

Then the circumstances of the decision problem will not change if it assumes in a natural way that we carry all sets of equipment in a rescue vehicle, but the vector function of the criterion function will change its form to:

$$
F_{1}(X)=\sum_{i=1}^{I} f_{i} \sum_{j=1}^{J} \sum_{k \in N^{i}}^{K} x_{j k} \cdot c^{k} \cdot w^{j}
$$

means the total weight of vertical displacement of rescue and firefighting equipment during various types of action,

$$
F_{2}(X)=\sum_{i=1}^{I} f_{i} \sum_{j=1}^{J} \sum_{k \in N^{i}}^{K} x_{j k} \cdot c^{k} \cdot d^{k}
$$

means the total weight of the horizontal displacement of rescue and firefighting equipment in the course of actions of different types, 


$$
F_{3}(X)=\sum_{i=1}^{I} f_{i} \sum_{j=1}^{J} \sum_{k \in N^{i}}^{K} x_{j k} \cdot \sum_{n \in Z_{k}} t_{n}
$$

means the total weighted time of movement of rescue and firefighting equipment during various types of action.

Then the task of multi-criteria optimization of the choice of distribution of rescue and firefighting equipment in a rescue and extinguishing vehicle will take the form of:

To choose such $\mathrm{X} *$ to minimize

$$
\begin{aligned}
& F_{1}^{\prime}\left(X^{*}\right)=\min _{X \in D} \sum_{i=1}^{I} f_{i} \sum_{j=1}^{J} \sum_{k \in N^{i}}^{K} x_{j k} \cdot c^{k} \cdot w^{j} \\
& F^{\prime}{ }_{2}\left(X^{*}\right)=\min _{X \in D} \sum_{i=1}^{I} f_{i} \sum_{j=1}^{J} \sum_{k \in N^{i}}^{K} x_{j k} \cdot c^{k} \cdot d^{k} \\
& F_{3}^{\prime}\left(X^{*}\right)=\min _{X \in D} \sum_{i=1}^{I} f_{i} \sum_{j=1}^{J} \sum_{k \in N^{i}}^{K} x_{j k} \cdot \sum_{n \in Z_{k}} t_{n}
\end{aligned}
$$

where the set $\mathrm{D}$ of acceptable solutions have the form:

$$
\begin{gathered}
x_{k j} \in\{0,1\}, k=1, \ldots, K, j=1, \ldots, J \\
\sum_{j=1}^{J} x_{k j}=1, k=1, \ldots, K \\
\sum_{k=1}^{K} x_{k j} \cdot r^{k} \leq p_{j}, j=1, \ldots, J \\
\left|\sum_{k=1,2,3} c^{k}-\sum_{k=4,5,6} c^{k}\right| \leq \varepsilon
\end{gathered}
$$

\section{The method for solving the problem}

Taking into account that:

- the number of solutions is not large,

- it can be effectively determined by different methods of solving multi-criterion linear optimization tasks,

- assuming that the vehicle manufacturer or equipment designer does not allow for different types of placement,

this is another approach: a series of simulation experiments can be performed (using the resulting vehicle simulator and capabilities in for example VBS3 environment [8] to simulate crew operations during fire or rescue operations using vehicle equipment) to investigate whether certain sequences of crew operations with fixed deployment kits on fire brigade vehicles and sequences of activities in the various types of rescue and firefighting operations are designed to provide the greatest ergonomics and crew safety during their operations.

In simulation experiments a sequence of actions will be performed:

- we choose another type of rescue and firefighting action,
- for a selected type of rescue and firefighting operation, we perform a series of experiments to evaluate

- the total weight of vertical displacement of lifefire extinguisher during such type of action,

- the total weight of horizontal displacement of lifefire extinguisher during such type of action,

- total time of moving firefighting equipment during firefighting such actions,

- we calculate

- the total weight of vertical displacement of lifefire extinguisher during such type of action according to the formula (32),

- the total weight of the horizontal displacement of rescue during such type of action according to the formula (33),

- total time of moving firefighting equipment during firefighting total time of moving firefighting equipment according to the formula (34),

- we choose (the selection method) the solution giving the best estimation of the criterion functions,

- decision-makers choose the solution that will suit them to the highest degree.

\section{Conclusions}

The problem of fireman equipment deployment on fire truck was formulated. It is linear integer programming problem with many criterion functions. It is able to solve that problem, after its modification, using formal method, for example by Gomory cutting plane method. However, the task is large enough to propose suggestion that it is possible to obtain the solution by virtual simulation.

The new method would be based on the method of determining the approximate solutions of optimization problem, and in the virtual simulation environment these solutions would be verified.

\section{References}

1. M. Jasiulewicz-Kaczmarek, A. Saniuk, T. Nowicki, The maintenance management in the macroergonomics context, Advances in Social \& Occupational Ergonomics, in series: Advances in Intelligent Systems and Computing, vol. 487, 35-46, Springer International Publishing AG (2017)

2. T. Nowicki, R. Waszkowski, A. Saniuk, HumanComputer interaction in sanitary inspection simulation exercises, Advances in Ergonomics Modeling, Usability \& Special Populations, in series: Advances in Intelligent Systems and Computing, vol. 486, 245-254, Springer International Publishing AG (2017)

3. M. Kiedrowicz, T. Nowicki, R. Waszkowski, Z. Wesołowski, K. Worwa, Optimization of the Document Placement in the RFID Cabinet, EDP Science, MATEC Web of Conferences 76, 02001 DOI:10.1051/matecconf/20167602001 (2016) 
4. P. Brucker Scheduling algorithms. Springer, Berlin, Heidelberg (2007)

5. M.L. Pinedo, Scheduling. Theory, algorithms and Systems. Springer, New York, London, Heidelberg (2008)

6. M.L. Pinedo, Scheduling. Planning and scheduling in manufacturing and services. Springer, New York (2005)

7. T. Nowicki, Linear Mixed Equivalent of a Stochastic Scheduling Problem with Different Processors Journal of Military University of Technology, 4 (1994)

8. J. Koszela, R. Wantoch-Rekowski, Designing advanced vehicle trainers using virtual simulation technology, Journal of KONES, 22, 265-272 (2015)

9. M. Kiedrowicz, R. Waszkowski, Business rules automation standards in business process management systems, (in:) Information Management in Practice, (eds) B.F. Kubiak and J. Maślankowski, pp. 187-200, (2015).
10. R. Hoffmann, M. Kiedrowicz, J. Stanik, Risk management system as the basic paradigm of the information security management system in an organization, MATEC Web of Conferences 76, 04010, DOI: 10.1051/matecconf $/ 20167604010$ (2016).

11. R. Hoffmann, M. Kiedrowicz, J. Stanik, Evaluation of information safety as an element of improving the organization's safety management, MATEC Web of Conferences 76, 04011, DOI:10.1051/ matecconf/20167604011 (2016).

12. M. Kiedrowicz, Location with the use of the RFID and GPS technologies - opportunities and threats, GIS ODYSSEY 2016, pp. 122-128, (2016).

13. M. Kiedrowicz, Objects identification in the informations models used by information systems, GIS ODYSSEY 2016, pp. 129-136, (2016).

14. M. Kiedrowicz, T. Nowicki, R. Waszkowski, Business process data flow between automated and human tasks, 3rd International Conference on Social Science (ICSS 2016) December 9-11 2016, pp. 471-477, (2016). 